\title{
Appropriate arrangement of cancer treatment after COVID-19 epidemic peaks in China
}

\author{
Hongnan $\mathrm{Mo}^{1} \cdot$ Binliang Liu ${ }^{1} \cdot$ Fei Ma ${ }^{1}$
}

Received: 10 May 2020 / Accepted: 25 May 2020 / Published online: 30 May 2020

c) Springer-Verlag GmbH Germany, part of Springer Nature 2020

\begin{abstract}
Purpose COVID-19 is causing a lot of problems in health services around the world, especially in medical institutions receiving cancer patients. On March 12, China's National Health Commission announced that the peak of the COVID-19 epidemic has passed in China. Thus, a proper arrangement of medication, surgery and radiotherapy for patients with cancer is of vital importance after the epidemic peak.

Methods A range of measures have been implemented in our center. Specific patients take priority for chemotherapy treatment. The amount of semi-elective and elective surgeries could be gradually increased beyond urgent and emergency surgery. The hypofractionated radiotherapy is recommended in the right circumstances.

Results On March 13, our center announced that more than 5000 visits of chemotherapy and radiotherapy are arranged in our outpatient clinics and none of our patients and staffs have been diagnosed with COVID-19 as of March 28, 2020.

Conclusion The rational arrangement we make now may be helpful to the future restoration of cancer treatments in other countries.
\end{abstract}

Keyword COVID-19 $\cdot$ Patient arrangement $\cdot$ Chemotherapy $\cdot$ Surgery $\cdot$ Radiotherapy

Dear Editor,

The novel coronavirus disease (COVID-19) has been characterized as a pandemic by World Health Organization on March 11, 2020 (World Health Organization 2020). Since the immune-suppressive nature of cancer treatments, as well as the frequent hospital visits, cancers patients have a higher risk to getting COVID-19 and will have a poorer outcome compared to the general population (Jing et al. 2020; Liang et al. 2020). Most of cancer institutes, including National Cancer Center/Cancer Hospital, Chinese Academy of Medical Sciences in China, have postponed non-urgent appointments. A longer delay or an interruption of cancer treatment inevitably increases the risk of tumor progression.

Hongnan Mo and Binliang Liu contributed equally to this work.

Fei Ma

drmafei@126.com

1 Department of Medical Oncology, National Cancer Center/ National Clinical Research Center for Cancer/Cancer Hospital, Chinese Academy of Medical Sciences and Peking Union Medical College, No. 17 Panjiayuan Nanli, Chaoyang District, Beijing 100021, China
China has been experiencing the outbreak of COVID-19 since December 2019. Drastic control measures substantially mitigated the spread of COVID-19 in China (Kraemer et al. 2020). On March 12, China's National Health Commission announced that the peak of the COVID-19 epidemic has passed in China. Thus, a proper arrangement of medication, surgery and radiotherapy for patients with cancer is of vital importance after the epidemic peak. A range of measures have been implemented in our center.

First, patients who underwent curative treatments, or those with a high recurrent risk, and had well tolerant to the treatment, take priority for intravenous medication. Chemotherapy regimens could be simplified to reduce recurrent visits. Outpatient treatment is more conducive to reducing crowd aggregation than inpatient treatment.

Second, the amount of semi-elective and elective surgeries could be gradually increased beyond urgent and emergency surgery. Some factors should be taken into consideration during this special period, such as the storage of protective gears, the availability of postoperative ICU, and the blood supply.

Third, patients with rapidly proliferating tumors like head and neck cancer and certain lymphomas should have the 
priority of taking radiotherapy. The hypofractionated radiotherapy is recommended in the right circumstances, because it may have similar efficacy and side-effects as a conventional fractionated radiotherapy and it is more convenient (Wang et al. 2019).

Clinical decisions should be discussed case by case to make sure the benefit gained from the continuous cancer treatments outweighs the risk of getting COVID-19. On March 13, our center announced that more than 5000 visits of chemotherapy and radiotherapy are arranged in our outpatient clinics and none of our patients and staffs have been diagnosed with COVID-19 as of March 28, 2020. The rational arrangement we make now may be helpful to the future restoration of cancer treatments in other countries.

Funding None.

Data availability The datasets generated during and/or analysed during the current study are available from the corresponding author on reasonable request.

\section{Compliance with ethical standards}

Conflict of interest The authors declare that they have no conflict of interest.

\section{References}

Kraemer M, Yang CH, Gutierrez B et al (2020) The effect of human mobility and control measures on the COVID-19 epidemic in China. Science. https://doi.org/10.1126/science.abb4218

Liang WH, Guan WJ, Chen RC et al (2020) Cancer patients in SARSCoV-2 infection: a nationwide analysis in China. Lancet Oncol 21(3):335-337. https://doi.org/10.1016/S1470-2045(20)30096-6

Wang SL, Fang H, Song YW et al (2019) Hypofractionated versus conventional fractionated postmastectomy radiotherapy for patients with high-risk breast cancer: a randomised, non-inferiority, openlabel, phase 3 trial. Lancet Oncol 20(3):352-360. https://doi. org/10.1016/S1470-2045(18)30813-1

World Health Organization (2020) Coronavirus disease (COVID-19) pandemic. https://www.who.int/emergencies/diseases/novel-coron avirus-2019. Accessed 28 Mar 2020

Yu J, Ouyang W, Melvin LK et al (2020) SARS-CoV-2 transmission in patients with cancer at a tertiary care hospital in Wuhan, China. JAMA Oncol. https://doi.org/10.1001/jamaoncol.2020.0980

Publisher's Note Springer Nature remains neutral with regard to jurisdictional claims in published maps and institutional affiliations. 